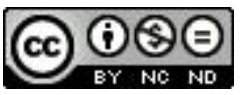

\title{
Construcción Social del Conocimiento Matemático durante la Obtención de Genes en una Práctica Toxicológica
}

\section{Social Construction of Mathematical Knowledge during the Isolation of Genes in a Toxicological Practice}

\author{
Isabel Tuyub Sánchez* \\ Ricardo Cantoral**
}

\section{Resumen}

Esta investigación centra su interés en el estudio de las prácticas sociales en la construcción del conocimiento matemático. Abandona la tradicional centración en el estudio de los conceptos matemáticos explícitos para encontrarlos en el ambiente científico de un toxicólogo que los pone en funcionamiento implícitamente. Se utiliza el constructo que temporalmente llamamos juego de prácticas, con la intención de analizar cómo construyen conocimiento innovador mediante procesos de institucionalización de las prácticas; tomando a la práctica social como generadora del conocimiento y reconociendo la importancia de los saberes en uso. Se propone un modelo en el que creencias, conocimientos, experiencia, expectativas, Representaciones Sociales y concepciones, juegan un papel en dicha construcción. Los resultados obtenidos son relativos al carácter funcional de la matemática en tanto herramienta para la construcción de conocimiento, el mecanismo localizado de optimización - estandarización tuvo la función normativa de la práctica estudiada.

\footnotetext{
" Maestra en Ciencias con especialidad en Matemática Educativa por el Centro de Investigaciones y de Estudios Avanzados del Instituto Politécnico Nacional (Cinvestav-IPN). Catedrática de la Facultad de Matemáticas de la Universidad Autónoma de Yucatán (FMAT-UADY), Mérida, Yucatán, México. Dirección del correo postal: anillo periférico norte, tablaje cat. 13615, colonia Chuburná Hidalgo Inn, Mérida, Yucatán, México.E-mail: isabel.tuyub@uady.mx.

*** Doctor en Ciencias con especialidad en Matemática Educativa por el Centro de Investigación y de Estudios Avanzados del Instituto Politécnico Nacional (Cinvestav-IPN), Departamento de Matemática Educativa. Jefe del departamento de Matemática Educativa del Cinvestav-IPN. Ciudad de México, México. Dirección del correo postal: Avenida Instituto Politécnico Nacional 2508, CP 07360. colonia San Pedro Zacatenco, Ciudad de México, México. E-mail: rcantor@cinvestav.mx.
} 
Palabras-clave: Socioepistemología de las Matemáticas. Prácticas Sociales. Procesos de Institucionalización. Matemáticas en la Actividad Científica de un Toxicólogo.

\begin{abstract}
This research report focuses on the study of the social practices in the construction of mathematical knowledge. Unlike the traditional focus on the study of explicit mathematical concepts, this study focused on the presence of mathematical concepts in the scientific atmosphere of a toxicologist, where they are used implicitly. We employ a construct that we will temporarily refer to as "sets of practices" to analyze how they construct innovative knowledge by means of processes of institutionalization of practices; considering that social practices are the generator of knowledge, and recognizing the importance of "knowledge in use". We propose a model in which beliefs, knowledge, experience, expectations, social representations, and conceptions play a role in this construction. The results are relative to the functional nature of mathematics as a tool for building knowledge. The localized optimization-standardization mechanism played a normative role in the practice studied.
\end{abstract}

Keywords: Socioepistemology of Mathematics. Social Practices. Process of Institucionalization. Mathematics in the Scientific Activity of a Toxicologist.

\title{
1 Introducción
}

Los procesos de enseñanza y aprendizaje en la escuela no logran que la matemática se torne funcional para la vida cotidiana de los ciudadanos. Esto ha sido caracterizado y documentado en diversos estudios (CANTORAL, 1990; CORDERO, 2005; BORELLO; FARFÁN; LEZAMA, 2008; CRESPO; FARFÁN; LEZAMA, 2010; PISA, 2009) en los que se afirma que los modelos conceptuales para el conocimiento matemático escolar están anclados al dominio teórico de la matemática pura y a una estructuración de conceptos aislados en la matemática escolar.

Investigaciones en el campo de la Matemática Educativa analizan la tesis que sostiene que el problema mayor de la enseñanza de las matemáticas no está en la organización y jerarquización temática del contenido, sino en la escasa claridad de la textura social del conocimiento matemático. Para entender las causas del fracaso escolar en matemáticas se debe considerar entonces, al mismo tiempo que al contenido, al conjunto de elementos institucionalizados que expresan una construcción social con sentido para el que aprende; estos saberes 
matemáticos deben su existencia, no a los motivos de su enseñanza, sino al hecho de haber estado al servicio de otros dominios científicos y de otras prácticas de referencia (FARFÁN, 1997; CANTORAL; FARFÁN, 1998).

Esta investigación asume, como problemática fundamental, al estudio de los mecanismos que permiten la construcción social del conocimiento, tomando en cuenta principalmente al saber en uso. Aspira con ello a entender, de mejor manera, los procesos de aprendizaje y aportar un modelo para la socialización de una visión científica del mundo entre la población.

Insistiremos, a lo largo de este escrito, en la construcción social de conocimiento matemático postulado como el programa Socioepistemológico impulsado por R. Cantoral. En dicho programa, se enfatiza la necesidad de estudiar diferentes escenarios de construcción del conocimiento sin prestar demasiada atención a los propios objetos matemáticos institucionalizados (número, conjunto, función, límite, derivada...) y prefiere poner cuidado al juego de prácticas desde donde dichos objetos emergen.

\section{La problemática}

La comunidad de Matemática Educativa ha estudiado la formación de nociones matemáticas, a fin de determinar cómo podrían ser mejor enseñadas y aprendidas (véase por ejemplo APOS Theory). En contraparte, se considera que no basta el estudiar cómo un individuo aprende algún concepto, sino se debe plantear el reto de caracterizar cómo es que lo socializa entre su comunidad, o más ampliamente, entre los ciudadanos. Es así que nos preguntamos si existe una manera matemática de pensar para que pueda ser difundida y compartida socialmente, cómo construimos nuestros sistemas conceptuales, cómo los institucionalizamos. Si bien estas interrogantes no pueden responderse directamente con nuestra investigación, en cambio abre una brecha en esa dirección.

Adicionalmente, en este programa, se cree que las respuestas a esas interrogantes no se encuentran en el estudio de la formación de conceptos aislados, sino en el análisis del juego de prácticas que lo acompaña; se precisa de estudios que toman en cuenta este hecho. Algunos son enfocados según la tesis de la génesis instrumental (BRISEÑO, 2008), los que estudian prácticas que transcienden culturalmente y que posibilitan una construcción social de conocimiento matemático (COVIÁN, 2005; CANUL, 2007; SIERRA, 2008; DE LIMA; MONTEIRO, 2009; CORDERO; CEN; SUÁREZ, 2010) o los que 
estudian prácticas en un ambiente científico (GARCÍA, 2008; GARCÍATORRES, 2008; TUYUB, 2008), pues antes de que los conocimientos emerjan, debe haber un contexto de significación y un escenario para la acción (CRESPO; FARFÁN; LEZAMA, 2009).

La presente investigación pretende comprender cómo cierto conocimiento se constituye como tal, por lo que analizamos aquellos mecanismos que posibilitan construirlo a través de procesos de institucionalización de las prácticas y el papel que tiene cierto saber en uso. Al realizar este cambio de focalización a las prácticas que permitan la constitución del conocimiento, se espera que pueda reflejarse con otros estudios en y hacia el aprendizaje de los alumnos, se busca innovar en la forma y tipo de investigación que realiza la Matemática Educativa, cuya metodología sea experimental.

Para ello, formulamos un modelo al seno de la Socioepistemología, referente al cómo se construye socialmente el conocimiento que se apoya fuertemente en la articulación de elementos cognitivos, sociales del saber; dicho modelo toma de referencia que las prácticas sociales ${ }^{1}$ son la base del conocimiento (CANTORAL; MÉNDEZ, 2009), una de las hipótesis de este programa; resaltando, de esta forma, su función normativa evidenciada en los procesos de institucionalización (CANTORAL, 1990; COVIÁN, 2005). Entonces, queda la cuestión teórica abierta de cómo se produce dicha normativa en dichos procesos cuando una comunidad produce conocimiento.

Esta pregunta condujo a la selección de una comunidad que realizase un juego de prácticas en el sentido que hemos descrito anteriormente, en la que se aprecie una articulación de sus actividades cotidianas, una organización de prácticas al seno de una práctica de referencia, normada por la práctica social.

Por ello, consideramos una comunidad que ejerza una práctica especializada en el campo de las ciencias, que produzca conocimiento innovador en periodos de tiempo no tan largos; la cual no debería poseer matemática explícita, ya que el riesgo mayor consistía en que si se estudiara, por ejemplo, una práctica matemática específica o propia de la ingeniería, podríamos ser orillados a estudiar objetos matemáticos, y no la forma en que sus juegos de prácticas dan forma a la utilización y a la construcción del conocimiento. Fue así que optamos por analizar una práctica toxicológica de carácter cualitativo pues, a priori, no había conceptos matemáticos familiares al investigador y de

\footnotetext{
${ }^{1}$ Son elementos que permiten la construcción del conocimiento, no anclan la problemática al dominio matemático y abren un camino conveniente para hacer estudios del uso del conocimiento matemático y su desarrollo (CANTORAL; FARFÁN, 1998).
} 
este modo se permitiría la inferencia de las prácticas.

Por lo anterior, elegimos una comunidad de toxicólogos ${ }^{2}$ en áreas de producción de conocimiento vanguardista en México. Así, nuestra pregunta primordial fue ¿cómo construyen conocimiento innovador los toxicólogos?

\section{Marco teórico}

Algunas investigaciones estudian prácticas profesionales enfocándose en el papel que desempeñan los conceptos matemáticos (ROMO; OKTAÇ, 2007), otras más, analizan la abstracción matemática en el ambiente laboral de estudio y considerando supuestos de lo que pudiese ocurrir con respecto a qué conceptos matemáticos usan, cómo los usan y cómo los considera la población de estudio al realizar su trabajo cotidiano, (NOSS; HOYLES; POZZI, 2000; KENT; NOSS, 2001, 2002). Estudiar a las prácticas, en este sentido, nos conduciría a una centración en el objeto matemático, aun al analizar prácticas; nuestra investigación en cambio, tomó como marco teórico a la Socioepistemología, una epistemología que modela las prácticas sociales que le dan origen, son fuentes y explican la construcción del conocimiento matemático (CANTORAL, 1990; FARFÁN, 1997; CORDERO, 2003; CANTORAL; FARFÁN, 2003).

Una de las tesis de la Socioepistemología parte de la premisa de que las prácticas sociales son las generadoras del conocimiento matemático a través de los diversos procesos de institucionalización (CANTORAL, 1990; CORDERO 2006). Tal enfoque, obliga a formular epistemologías del conocimiento cuya centración está en su constitución social natural, en "lo" que hace que el conocimiento sea así y no de otra manera, reconoce al grupo humano en su organización, historia, cultura e institución que lo lleva a proceder de una manera y no de otra; su práctica social generatriz de su conocimiento (CANTORAL, 1990; FARFÁN, 1997; CORDERO, 2005). Se considera una teoría relativista en tanto que no busca principios o leyes absolutas, sino explicaciones al escenario.

Se eligió esta teoría por la peculiar forma en que considera a las prácticas sociales, no ancla su objeto ni su problemática de investigación al dominio específicamente matemático, pues sus elementos permiten la construcción del conocimiento en un sentido social más amplio y abonan a un camino para estudiar los usos del conocimiento matemático y su desarrollo al fomentar una centración

\footnotetext{
${ }^{2}$ Científicos que estudian los efectos adverso de sustancias químicas en organismos vivos.
} 
en aquellas prácticas que permiten la construcción de dicho conocimiento (CANTORAL; FARFÁN, 2003; FARFÁN; FERRARI, 2008; OLIVEIRA; ZOCH; RYOKITI, 2009).

Sin embargo, debido a que el estudio requería de elementos de naturaleza social, se consideró como apoyo de ciertos constructos teóricos, que no nacieron dentro de la Matemática Educativa, pero son un gran apoyo para explicar fenómenos dentro de ella, como son las representaciones sociales (MOSCOVICCI, 2003). Las cuales se definen como formas de conocimiento laborado socialmente y compartido con un objeto práctico, que concurre bajo la construcción de una realidad común para un conjunto social, es un proceso mediante el cual explican su realidad, la cubren de elementos afectivos y le dan un significado coherente en su estructura de pensamiento, está socialmente marcada; se producen, se recrean y se modifican en el curso de las interacciones y prácticas (MOSCOVICCI, 1998 apud CASTORINA; KAPLAN, 2003).

\section{Modelo Teórico de Construcción (MTC)}

El MTC se generó como un medio de análisis del juego de prácticas. Ello ocurre al seno de una comunidad de socioepistemólogos que considera al aprendizaje como cambio de prácticas. Este modelo parte de la explicación sobre la construcción del conocimiento de acuerdo a la función normativa de la práctica social, enfatiza la descentración de los conceptos, considerando al saber en la práctica; un antecedente se encuentra en (TUYUB; CANTORAL, 2007).

En la Figura 1 se observa el esquema del MTC: parte del supuesto teórico de la existencia de prácticas sociales como bases del conocimiento, con la intención de no permitir una regresión infinita de prácticas iniciales. Se aprecia la relación que pueden tejerse entre prácticas, experiencia, socialización, conocimientos, creencias, expectativas, concepciones y Representaciones Sociales, aunado a un saber en uso, situado en un contexto social, cultural, histórico e institucional. Considerar al saber, enfocándose en prácticas y no en conceptos matemáticos, permite al modelo no limitarse al plano cognitivo. 


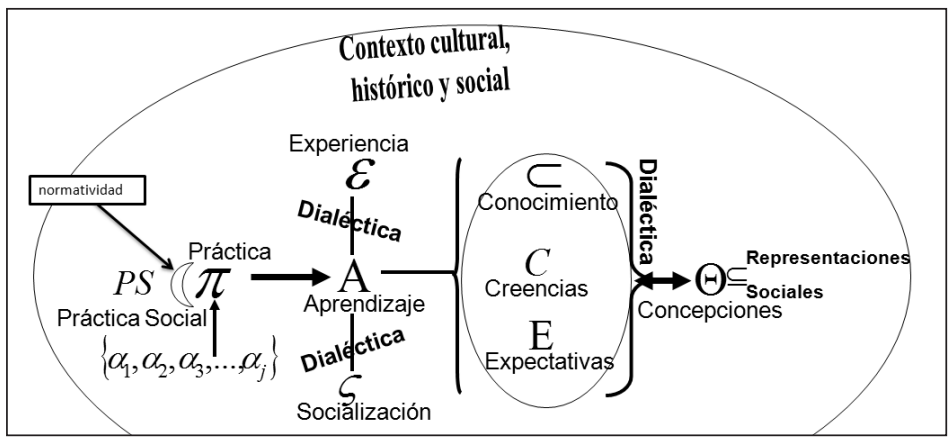

Figura 1 - Modelo Teórico de Construcción, en el que se involucran ciertos elementos cognitivos y sociales necesarios para la construcción de conocimiento inmerso en cierto contexto. Este modelo busca explicar, sintéticamente la forma en que las concepciones se incluyen en las representaciones sociales y cómo ambas se guían por la función normativa de la práctica social

\section{Metodología}

Nos interesa transitar de la descripción de los actos de entendimiento de un individuo, a la comprensión de los motivos por los cuales la comunidad elegida ejerce ciertas prácticas. Se parte así de una actividad que organizada y sistematizada deviene en una práctica, la cual ocurre en el contexto de una práctica de referencia más sofisticada y que, articulada con otras prácticas de referencia, permite entender las normativas que la regulan. Es a esta función normativa de la práctica a la que atribuiremos la estructura comprensiva del juego de prácticas.

Para efectos del estudio, utilizamos una metodología cualitativa de corte etnográfico, a fin de inferir las prácticas de una comunidad de toxicólogos especialistas en los efectos de los pesticidas sobre la reproducción masculina, por medio del quehacer cotidiano de $\mathrm{M}$, un investigador en etapa de entrenamiento al nivel posdoctoral en el área de Biología Molecular (grado obtenido después de realizar un doctorado en ciencias en la especialidad de Genética). Se observaron los momentos en que $\mathrm{M}$ realizaba un protocolo ${ }^{3}$ nuevo sobre la identificación de genes inmunes al cáncer del próstata (T1 y M1) en el ADN. Genes de personas expuestas a pesticidas, que resultan significativos para identificar qué porcentaje de la población los poseen, en una comunidad

\footnotetext{
${ }^{3}$ Documentos en los que redactan un conjunto sistematizado de pasos a seguir para realizar experimentos de sus investigaciones, y que serán imprescindibles para utilizar en experimentos futuros para los demás colegas de su comunidad científica.
} 
campesina.

Si bien es cierto que analizar el juego de prácticas de una persona no basta para inferir sus alcances al nivel de una comunidad, consideramos que analizar en profundidad el juego de prácticas de una persona situada en un escenario, permite realizar un estudio de la comunidad y sus hábitos, pues su hacer no depende de la experiencia individual, sino de cierto conocimiento y expectativas puestas en juego compartidas por la comunidad.

Para la recolección de información empleamos la observación participante, entrevistas y grabaciones de las actividades de $\mathrm{M}$ en el proyecto de los genes, así como las descripciones y registros de las interacciones en escenas claves, en escenarios específicos de su práctica científica.

Fue indispensable para nuestro propósito el contar con una caracterización de la población y de la comunidad a la que pertenecen, así como un análisis de corte epistemológico del quehacer en Toxicología - en tanto área tecnológica a fin de localizar y aislar las prácticas de referencia, en el sentido de Farfán $(1997,2003)$ con la intención de identificar y relacionarnos con el contexto. Por ello, durante un año de registro cotidiano y de inmersión en su comunidad, se intentó localizar mecanismos en las que se podía inferir a través un proceso de institucionalización de sus prácticas y del papel que tiene el saber en uso.

Para el análisis de los datos recolectados realizamos un corte directo del video para la obtención selectiva de momentos clave por medio de tres ejes de análisis, que serán descritos a continuación. Nos apoyamos en las transcripciones de las interacciones y en las notas campo que levantamos durante las intervenciones cotidianas.

\section{Análisis de resultados}

Se consideraron tres ejes de análisis: identificación de prácticas significativas, dentro de ellas, actividades asociadas a un saber funcional y, a la par, identificación de procesos de institucionalización en dichas prácticas.

Eje 1. Análisis de prácticas significativas que giran en torno de un objeto toxicológico (en nuestro caso el ADN), con el fin de elegir aquellas que proporcionan datos a través de qué es lo que las regula (las denominamos prácticas asociadas).

Eje 2. Identificación de actividades asociadas a un saber funcional, en particular asociados al uso de la función matemática (que denominamos functilidad).

Eje 3. Identificación de procesos de institucionalización en las prácticas 
asociadas, mediante la dialéctica de permanencia y cambio en la toma de decisiones de M. Con este eje deseamos determinar el sentido normativo de las prácticas sociales.

En la articulación de dichos ejes es donde se identificaron los mecanismos de construcción de conocimiento.

A través del eje 1 identificamos tres prácticas asociadas: obtención de ADN a partir de tejidos, amplificación de los genes M1 y T1 por PCR (Reacción en Cadena de la Polimerasa) y monitoreo de éstos en el ADN (Análisis de fotos). Organizadas para permitir la obtención del protocolo, en las cuales analizamos los ejes 2 y 3 para obtener la información de nuestra investigación.

Este eje permitió obtener un modelo experimental dinámico sobre la organización de las prácticas asociadas. En la Figura 2, se aprecia el esquema de lo identificado experimentalmente. Las flechas señalan el dinamismo de la práctica de $\mathrm{M}$.

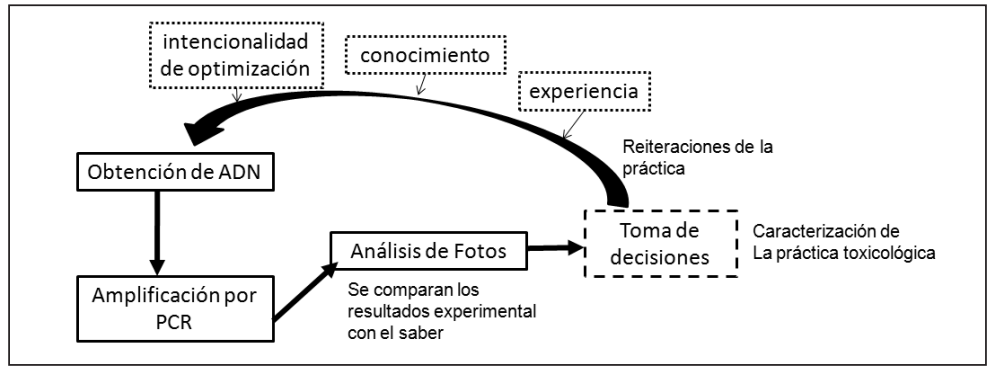

Figura 2 - Esquema obtenido de estudiar prácticas asociadas para caracterizar la práctica toxicológica

Este esquema fue apreciado en la toma de decisiones de M, admitiendo a la caracterización de su práctica toxicológica. Es decir, en las prácticas asociadas pudimos inferir aspectos como la experiencia, el conocimiento, inmersos en el MTC que afectaban la práctica de M, cuando repetía nuevamente el experimento porque no obtuvo los resultados deseados, al momento de comparar lo que obtenía experimentalmente con lo estipulado por su comunidad como aceptable.

Otro elemento identificado, no contemplado en el MTC, es la intencionalidad para optimizar tiempo, esfuerzo y recursos, como una de las necesidades para cambiar sus prácticas.

A continuación mostraremos el análisis y resultados obtenidos de los ejes 2 y 3, que se analizaron de manera transversal en cada una de las tres prácticas asociadas. 
Con respecto al eje 2, M realizó cambios al momento de remplazar una actividad por otra, cuando utilizó el trizol para homogenizar, en lugar del homogenizador, que es lo que se marcaba en un libro de toxicología, la razón se comenta en el fragmento 1. M: Te acuerdas que esto lo hacía yo con el ¡homogenizador! Y, ¿sí?, bueno, ya si te has fijado que esto lo he ido [habla como si se quiere reír] optimizado ¿sí? [Silencio];No! y aparte ¡la baba! y cosas así [risas] [silencio], y yo veía que la pastilla era jmuy poquita! lo que obtenía (Fragmento 1 - Sesión 11. 6’20”-6’48”).

Este cambio introducido por M, fue en pro para la obtención del ADN, de tal forma que se obtenga lo más que se pueda del producto, esta optimización establece una relación de dependencia, elemento esencial de la functilidad.

En el fragmento 2, M agrega una sustancia al ADN y expresa que la coloca poco a poquito, para no degradarlo, en ese momento manifiesta un conocimiento sobre flujo, este es otro ejemplo de functilidad, cuya acción se aprecia en la Figura 3. M: Si te fijas, o sea, cada vez que estoy haciendo esto, estoy haciendo cosas nuevas, optimizo ¿sí? Antes de, por ejemplo ahorita con este, no le voy a echar todo el trizol porque luego batallo para re suspenderlo, se lo voy a ir poniendo poco a poquito, que si viste que ahorita se lo eché todo y estuve batalle y batalle (Fragmento 2 Sesión 14. 73' 35" - 74'02").

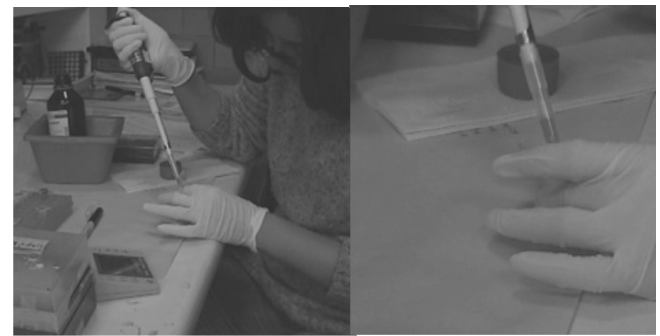

Figura 3 - Se observa a M usando una pipeta con una sustancia llamada trizol para homogenizar el tejido poco a poco, actividad a la que se refiere el fragmento 3

Con base en la toma de decisiones se van realizando cambios, por ello la obtención de $\mathrm{ADN}$ en la práctica no es lineal, sino presenta bifurcaciones donde se aprecia qué actividades se dejaron de hacer o cuáles se cambiaron por otras para cumplir el objetivo.

La Figura 4 exhibe un esquema de cómo son los cambios realizados en virtud de la toma de decisiones en las reiteraciones, en ella se muestra la no linealidad de la práctica de obtención de ADN 


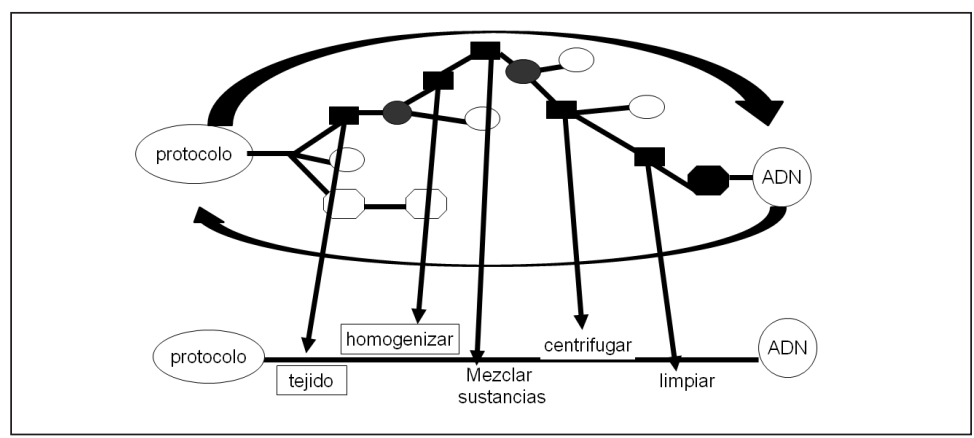

Figura 4 - Esquema de la práctica asociada obtención de $A D N$, inferido de sus reiteraciones. Muestra la organización de las actividades realizadas en la toma de decisiones de $\mathrm{M}$ al momento de realizar la práctica

Con base en lo anterior, la práctica de $\mathrm{M}$ puede inferirse como una función matemática de varias variables. La función será la práctica en sí y las actividades que la conforman serán las variables, donde tiene que decidir cuáles dejará constantes y cuáles variará para obtener los resultados de su investigación, como lo es obtener un ADN limpio y puro.

Una vez obtenido el ADN, M lo utiliza para realizar una PCR, que consiste en colocar ciertos reactivos con determinada medida en cada ADN y obtener una foto, como la que se muestra en la Figura 5, para ver si las cantidades que colocó son las deseadas y, si no, modificar algo más hasta que obtenga los resultados deseados (los que su comunidad reconoce).

A B C D E F G H I J

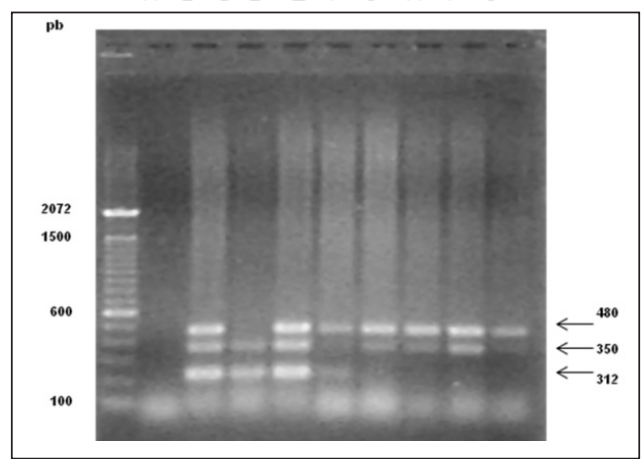

Figura 5 - Gráfica obtenida experimentalmente. Resultado de un proceso que involucra las prácticas asociadas. Las amplificaciones de los genes son las bandas definidas a ciertas alturas, la ausencia de bandas indica la no presencia de ese gen en el individuo estudiado 
Este análisis de fotos es, en realidad, otro uso del sentido de función matemática, reflejada en un análisis de gráficas en tres dimensiones: observando la Figura 5, eje de las $x$ contiene los ADNs distintos (A-J)), el eje de las $y$ la altura en peso molecular que puede alcanzar el gen en ese ADN (va de 100 en 100 pares de base) y el eje $z$ marcado por la intensidad de las bandas o segmentos definidos a determinada altura, los cuales son los genes amplificados.

Con respecto al eje 3, para la obtención de $\mathrm{ADN}$, M tenía que obtener primero tejidos de los órganos reproductivos de ratones; $\mathrm{M}$ comentaba que era la primera vez que realizaba esta práctica, para ello se basó en uno protocolo para extraer el ADN de otro tipo de tejidos, que estipulan los pasos a seguir a fin de desarrollar el experimento. Sin embargo, conforme a las repeticiones de su práctica, aumentó y cambió algunos procesos. Por ejemplo, utilizó tijeras para cortar tejidos de testículo de ratón, lo cual no está considerado en el protocolo que tomó de guía, en el Fragmento 3 de nuestros registros justifica la razón de su cambio: Entrevistador: ¿Por qué lo estás cortando? M: Porque te acuerdas el otro día, bueno, este es un tejido muy duro y...el otro día no lo recuperé, este, también como que no, hay que romperlo y hacerlo así...entonces para que se disuelva mucho más rápido cuando le ponga el trizol (Fragmento 3 Sesión 11. 5'42"-6'17"').

Si bien el protocolo que usó $\mathrm{M}$ de referencia era aceptado por su comunidad para otro tipo de tejidos menos delicados, al obtener resultados cercanos a los aceptados por su comunidad, como lo es que el ADN no esté contaminado o la cadena de ADN esté completa, sus prácticas eran más estables, es decir, casi no se apreciaban cambios en ellas.

Se infirieron elementos de permanencia y cambio a través de las reiteraciones de las prácticas asociadas, en las que intervinieron factores contemplados en el MTC, como la interacción con colegas, su conocimiento, expectativas, la presión que sentía cuando los resultados no salían, sus creencias y concepciones sobre el ADN, sus representaciones sociales sobre lo que es ser un posdoctor y la experiencia, entre otros; que permiten una toma de decisiones en pro de un resultado certero (determinación de la presencia de los genes T1 y M1). Al tratar de determinar estos elementos de permanencia y cambio, notamos que sólo eran fotografías de la evidencia, y, por tanto, la necesidad de obtener mecanismos que nos permitieran responder a qué se debían, con énfasis en los procesos.

Como se puede apreciar, los procesos de institucionalización rigen la práctica toxicológica de $\mathrm{M}$, cuando lleva a cabo experimentos con la intención de amplificar los genes como debe ser, como la comunidad los acepta, para la 
utilización de su protocolo; se manifiesta un debate entre el conocimiento teórico y el experimental, con la intencionalidad de obtención de los genes M1 y T1 de forma clara y con la menor cantidad de recursos, esfuerzo y tiempo (optimización).

Detectamos que algunos elementos que caracterizan la práctica de $\mathrm{M}$ están inmersos en el MTC, por ejemplo, se detectó un mecanismo de socialización entre sus colegas sobre cómo homogenizar de la mejor manera. La creencia de que los resultados no salían porque los ADNs tenían sales, la concepción de que los ADNs se encontraban en mal estado por su conocimiento previo, su experiencia, acerca del tiempo de congelación, la forma de extracción y conservación, la representación social que manifiesta $\mathrm{M}$ respecto al significado de ser un posdoctor ${ }^{4}$, como una persona independiente y experta, regulaba su práctica al actuar como tal, y a la vez eran factores que permitían la construcción de su conocimiento basado en saberes en uso y procesos de institucionalización.

Con base en los tres ejes analizados, un mecanismo de construcción que identificamos es la dupla optimización-estandarización, M optimiza (economiza tiempo, esfuerzo, recursos sin perder la calidad y certeza de sus datos) con la intención de estandarizar (tener resultados constantes sin importar quien realice la técnica por medio de los pasos de su protocolo). Es la dupla mecanismofunción (uso), base del mecanismo empleado por M: tubos, centrífuga, reactivos (instrumento), los emplea en sus experimentos de forma óptima (mecanismo) con base en su experiencia, conocimientos, creencias, concepciones y representaciones sociales con la intención de estandarizar (función).

\section{Consideraciones finales}

La forma en cómo se afecta la práctica de $\mathrm{M}$, es una manera de caracterizarla y es, a su vez, un signo visible para la determinación de esta afectación. Es por medio de la toma de decisiones apreciada en la reiteración de su práctica que esto se lleva a cabo, ya que es ahí donde identificamos los cambios.

Los tres ejes de análisis permitieron mirar desde distintos puntos de vista la práctica de $\mathrm{M}$ y dieron elementos para comprender las razones de por qué $\mathrm{M}$ hace lo que hace. Así mismo, permitieron estudiar factores no previstos en el MTC y de gran importancia para la construcción social de conocimiento, por ejemplo la presencia de los saberes en uso y los procesos de

\footnotetext{
${ }^{4}$ Porque $\mathrm{M}$ comentaba que no era un estudiante y que no debía tener a nadie que le revisara sus datos de sus investigaciones, y actuaba como tal, de forma independiente, es decir su RS, en cierta forma, regulaba su práctica.
} 
institucionalización no expresados puntualmente en el modelo, pero presentes al momento de estudiar la práctica toxicológica de $\mathrm{M}$.

Los elementos contemplados en el MTC fueron identificados en las reiteraciones, de ahí que se considera como modelo dinámico, en constante evolución, donde sus elementos interactúan y caracterizan la práctica de M. En la Figura 6 se aprecia el modelo robustecido.

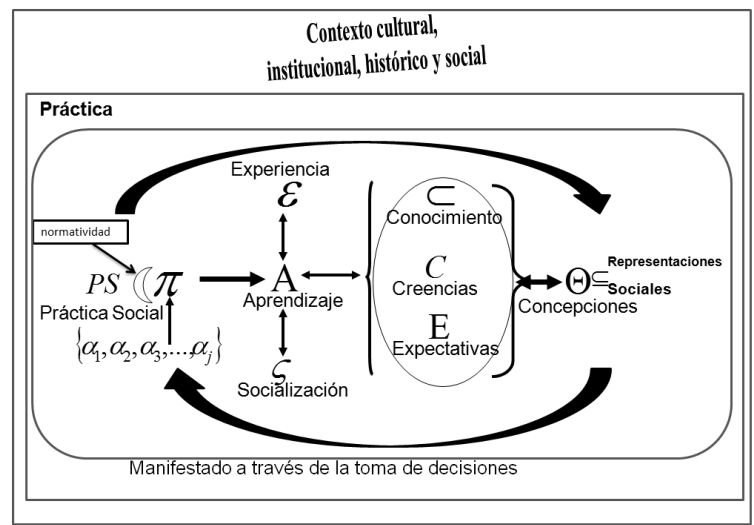

Figura 6 - Modelo Teórico robustecido por el análisis de datos

La functilidad fue herramienta para resolver problemas toxicológicos, aunque $\mathrm{M}$ no fue consciente de su uso; nos permitió entender la matematización de cualidades. Por ejemplo, el análisis de gráficas era el paso final para tomar decisiones, si los genes se obtenían borrosos, entonces era momento de realizar el experimento nuevamente, cambiando algunas variables, y para ello intervinieron conocimientos, expectativas, concepciones, creencias, entre otros.

Al estudiar la construcción de un nuevo protocolo, apreciamos los procesos de institucionalización y por ende evidenciamos la función normativa de la práctica social en el por qué de las modificaciones y permanencias, inferidos en la continuidad de las prácticas, sobre qué es lo que las permite, apoyado de mecanismos de construcción; con lo que obtenemos datos para seguir estudiándolos y considerarlos en la educación de las matemáticas y darle a esta ciencia un carácter funcional.

En la toma de decisiones de M, inferimos procesos de institucionalización, originados para resolver un problema no contemplado, obtener resultados esperados y optimizar (tiempo, material y esfuerzo) con intención es estandarizar (obtener un protocolo que permita la determinación de los genes en ADN), el mecanismo identificado fue optimización-estandarización, que en términos 
socioepistemológicos se refiere a la dupla mecanismo-intencionalidad (en el sentido de su uso).

Al estudiar las prácticas, para obtener la evidencia y los datos, no basta con atender al discurso explícito, se requiere, además de los elementos no verbales, típicamente situacionales, y los que están en el discurso pero no se perciben como evidencia del conocimiento matemático en juego: como cuando M menciona (en el fragmento 3): coloco el trizol ahora poco a poquito para no batallar en homogenizar el tejido; el término poco a poquito identifica el concepto funcional de flujo.

Si bien nuestra investigación se lleva a cabo en un escenario toxicológico, no aportamos a dicha área del conocimiento, sino se trata más bien de un intento por comprender a profundidad cómo es que construyen socialmente el conocimiento. Al estudiar el juego de prácticas de esta comunidad mediante el análisis del quehacer de M, no sólo se está prestando atención a sus acciones, sino al fundamento que orienta sus prácticas, el saber las razones por las que lo hacen de ese modo y no de otro.

Esta investigación ofrece una brecha novedosa para atender la descentración de los conceptos y brinda elementos metodológicos para el estudio de prácticas en una forma continua. En consecuencia, amplía el panorama de la problemática abordada en la Matemática Educativa al no mirar sólo a los conceptos (institucionalizados y externos al individuo) sino en las prácticas; no basta el aprendizaje (en el sentido cognitivo) para apropiarse de elementos matemáticos, se debe lograr que las personas vivan experiencias a través de cambio de prácticas ante determinado problema, que infieran procesos y tomen en cuenta el sentido funcional del conocimiento matemático. El uso situacional antes que el significado institucional.

\section{Referencias}

BORELLO, M.; FARFÁN, R.; LEZAMA, J., Relazione tra le concezione e le idee del docente e l'apprendimento dell'allievo nel caso delle disequazioni: lo estato dell'arte. La Matemática e la sua Didattica, Bolonia, Italia, v. 223, n. 3, p. 331 - 361, 2008.

BRISEÑO, E. El uso de las gráficas desde una perspectiva instrumental. Un estudio socioepistemológico. 2008, 141f. Tesis (Maestría en Matemática Educativa) - Centro de Investigaciones y Estudios Avanzados, Instituto Politécnico Nacional, Ciudad de México, México, 2008. 
CANTORAL, R. Categorías Relativas a la apropiación de una base de significaciones para conceptos y procesos matemáticos de la Teoría elemental de las Funciones

Analíticas: simbiosis y Predación entre las nociones de "el Prædiciere y lo Analítico". 1990, 554f. Tesis (Doctorado en Matemática Educativa) - Centro de Investigaciones y Estudios Avanzados, Instituto Politécnico Nacional, Ciudad de México, México, 1990.

CANTORAL, R.; FARFÁN, R. Pensamiento y lenguaje variacional en la introducción al análisis. Epsilon, Andalucía, España, v. 42, n.3, p. 353 - 369, 1998.

CANTORAL, R.; FARFÁN, R. Mathematics Education: a vision of its evolution. Educational Studies in Mathematics. Netherlands, Kluwer Academic Publishers, Issue, v. 53, n.3, p. 255 - 270, 2003.

CANTORAL, R.; MÉNDEZ, M. Introducción: “aspectos socioepistemológicos en el análisis y el rediseño del discurso matemático escolar". In: REUNIÓN LATINOAMERICANA DE MATEMÁTICA EDUCATIVA, 22., 2008, Ciudad de México, México. Acta... Ciudad de México: Clame, 2009, p. 1055 - 1060.

CANUL, G. La práctica del bordado como generadora de conocimiento matemático. 2007. 138f. Tesis (Maestría en Matemática Educativa) - Centro de Investigaciones y Estudios Avanzados, Instituto Politécnico Nacional, Ciudad de México, México, 2007.

CASTORINA, J.; KAPLAN, C. Representaciones sociales: problemas teóricos y desafíos educativos. In: CASTORINA, J. (Ed.). Representaciones sociales: problemas teóricos y conocimientos infantiles. Barcelona: Editorial Gedisa, 2003. p. 9 - 27.

CORDERO, F.; CEN, C; SUÁREZ, L. Los funcionamientos y formas de las gráficas en los libros de texto: una práctica institucional en el bachillerato. Revista Latinoamericana de Investigación en Matemática Educativa, Ciudad de México, México, v. 13, n. 2, p. 187 - 214, 2010.

CORDERO, F. Reconstrucción de significados del Cálculo Integral: la noción de acumulación como una argumentación. Ciudad de México, México: grupo Editorial Iberoamérica, 2003, 269p.

CORDERO, F. La distinción entre construcciones del cálculo: una epistemología a través de la actividad humana. Revista Latinoamericana de Investigación en Matemática Educativa, Ciudad de México, México, v. 4, n. 2, p. 103 - 128, 2005.

CORDERO, F. La institucionalización del conocimiento matemático y el rediseño del discurso matemático escolar. In REUNIÓN LATINOAMERICANA DE MATEMÁTICA EDUCATIVA, 19., 2006, Ciudad de México, México. Acta...Ciudad de México: Clame, 2006, p. 824 - 830. 
COVIÁN, O. El papel del conocimiento matemático en la construcción de la vivienda tradicional: el caso de la cultura Maya. 2005 192f. Tesis (Maestría en Matemática Educativa) - Centro de Investigaciones y Estudios Avanzados, Instituto Politécnico Nacional, Ciudad de México, México, 2005.

CRESPO, C.; FARFÁN, R.; LEZAMA, J. Algunas características de las argumentaciones y la matemática en escenarios sin influencia aristotélica. Revista Latinoamericana de Investigación en Matemática Educativa, Ciudad de México, México, v. 12, n. 1, p. 29 - 66, 2009.

CRESPO, C.; FARFÁN, R; LEZAMA, J. Argumentaciones y demostraciones: una visión de la influencia de los escenario socioculturales. Revista Latinoamericana de Investigación en Matemática Educativa, Ciudad de México, México, v. 13, n. 3, p. 283 $306,2010$.

DE LIMA, M.; MONTEIRO, A. Práticas Sociais de Localização e Mapeamento: uma discussão curricular sobre o conceito de escala. Bolema, Rio Claro, Brasil, Ano 22, n. 32, p. 1 - 28, abril. 2009.

FARFÁN, R. Ingeniería Didáctica y Matemática Educativa: un estudio de la variación y el cambio. México: Grupo Editorial Iberoamérica, 1997, 283p.

FARFÁN, R. Uma pesquisa em Educação Matemática: da propagação do calor à noção de convergência. Educação Matemática Pesquisa, Sao Paulo, Brasil, v. 5, n. 2, p. $39-58,2003$.

FARFÁN, R.; FERRARI, M. Un estudio socioepistemológico de lo logarítmico: la construcción de una red de modelos. Revista Latinoamericana de Investigación en Matemática Educativa Ciudad de México, México, v. 11, n. 3, p. 309 - 354, 2008.

GARCÍA, E. El uso del conocimiento matemático asociado a la función en la producción institucional: el caso de investigadores en formación en Matemática Educativa. 2008. 159f. Tesis (Maestría en Matemática Educativa) - Centro de Investigaciones y Estudios Avanzados, Instituto Politécnico Nacional, Ciudad de México, México, 2008.

GARCÍA-TORRES, E. Un estudio sobre los procesos de institucionalización de las prácticas en ingeniería biomédica: una visión socioepistemológica. 2008. 105f. Tesis (Maestría en Matemática Educativa) - Centro de Investigaciones y Estudios Avanzados, Instituto Politécnico Nacional, Ciudad de México, México, 2008.

KENT, P.; NOSS, R. Investigating the mathematical components of engineering expertise. In: PSYCHOLOGY OF MATHEMATICS EDUCATION (Conferencia), 25. 2001, Utrecht, Holanda. Proceeding... Utrecht, Holanda: IOE London, 2001. p. 402 415. Disponible en: <http://www.lkl.ac.uk/research/MCEE/MCEE-poster-forPME25.pdf >. Acceso en: 2 sept. 2008. 
KENT, P.; NOSS, R. The mathematical components of engineering expertise: the relationship between doing and understanding mathematics. En: SYMPOSIUM ON ENGINEERING EDUCATION, (Conferencia), 2., 2002. London, Uk. Proceeding... London, Uk: IOE London, 2002. p. 1 - 7 Disponible en: <http/k1.ioe.ac.uk/rnoss/ MCEE/MCEE-poster-for-PME25.pdf>. Acceso en: 2 sept. 2008.

MOSCOVICCI, S. La conciencia social y su historia. In: CASTORINA, J. (Ed.).

Representaciones sociales: problemas teóricos y conocimientos infantiles. Barcelona: Editorial Gedisa. 2003.p. 91 - 110.

NOSS, R.; HOYLES, C.; POZZI, S. Working Knowlegde: mathematics in use. In: BESSOT, A.; RIDGWAY, J. (Eds.). Education for Mathematics in workplace. Netherlands: Kluwer academic publishers, 2000, p. 17 - 35.

OLIVEIRA, C.; ZOCH, L.; RYOKITI, A. Sequiência Didática com Análise Combinatória no Padrão SCORM. Bolema, Rio Claro, Brasil, Ano 22, n. 34, p. 27 - 56, dez. 2009.

PROGRAMMEFOR INTERNATIONAL STUDENT ASSESSMENT- PISA/OCDE: Anual Report. 2009. Disponible em: <http://www.oecd.org/dataoecd/38/39/ 43125523.pdf>. Acceso en: 5 ago. 2009.

ROMO, A.; OKTAÇ, A. Herramienta metodológica para el análisis de los conceptos matemáticos en el ejercicio de la ingeniería. Revista Latinoamericana de Investigación en Matemática Educativa, Ciudad de México, México, v. 10, n. 1, p. 117 - 143, 2007.

SIERRA, E. Pesas y medidas: un estudio socioepistemológico: el caso Metlatónoc. 2008, 84f.Tesis (Maestría en Matemática Educativa) - Universidad Autónoma de Guerrero, Chilpancingo, Guerrero, México, 2008.

TUYUB, I. Un estudio socioepistemológico de la práctica toxicológica. Un modelo sobre la construcción social del conocimiento. 2008, 159f. Tesis (Maestria en Ciencias, especialidad en Matemática Educativa) - Centro de Investigaciones y Estudios Avanzados, Instituto Politécnico Nacional, Ciudad de México, México, 2008.

TUYUB, I.; CANTORAL, R. Las prácticas sociales como base del conocimiento en toxicólogos: un modelo. In: MONTIEL, G; BUENDIA, G. (Eds.). Memorias de la XI Escuela de Invierno en Matemática Educativa. Ciudad de México, México: Red Cimates, 2007, p. 141 - 153.

Submetido em Junho de 2010. Aprovado em Novembro de 2010. 